Journal of Engineering and Applied Sciences 15 (5): 1060-1066, 2020

ISSN: $1816-949 \mathrm{X}$

(C) Medwell Journals, 2020

\title{
Technique of Mathematical Simulation of Input Signals of Onboard Radars from Complex Scenes with Clutters and Targets
}

\author{
${ }^{1}$ Andrei Likhoedenko and ${ }^{2}$ Victor Suchkov \\ ${ }^{1}$ Higher School of Economics, Moscow, Russia \\ ${ }^{2}$ Department of Autonomous Information and Control Systems, \\ Bauman Moscow State Technical University, Moscow, Russia
}

\begin{abstract}
The problem of creation of complex radar scene with various objects are described. For decision of this problem the polygonal models of clutters and targets are used. It is shown that the polygonal models of targets could be formed in PTC Creo on the base of FEM mesh. In the other side, the polygonal models of clutters, like rough surfaces are easily to develop in 3DSMAX with use of noise modifier. With use of the suggested technology the trajectory's signals of onboard radars from various complex scenes are calculated on the base of polygonal and multipoint models. Investigation of characteristics of input signals from complex targets and clutters was carried out for such applications of onboard radars as drone radar and automotive radars. It is shown possibility of use of asymptotic diffraction methods like physical optics and physical theory of diffraction.
\end{abstract}

Key words: Mathematical simulation, diffraction, clutter, target, radar cross section, onboard radar

\section{INTRODUCTION}

The onboard short range radars often operate in the conditions of influence of the clutters. So, development of new techniques of calculation of input signals of radar detectors from clutters is necessary. Such systems as radio altimeters, measuring instruments of parameters of soil, use the signal reflected from the spreading surface as useful and take from him information on required characteristics.

Nowadays a lot of theoretical and empirical models of scattering fields from clutters of different kind are developed. Most theoretical methods are based on the solution of a classical problem of diffraction of an electromagnetic wave with use of Physical Optics (PO) or Method of Moments (MoM). Moreover, for digital simulation of specific Radar Cross Section (RCS) of clutters the capability of exact description of geometrical shape and electrodynamic parameters of surface are required. Existing empirical models of specific RCS are based on the analysis of experimental results obtained for different types of equipment and techniques. As a result, actual problem of mathematical simulation of input signals of onboard radars is creation accurate and fast models of clutter's scattering characteristics.

In the study, we would like to represent a technique and results of calculation of input signals of onboard radars from radar scenes with clutters on the basis of their multipoint models.

\section{MATERIALS AND METHODS}

Review of techniques of mathematical simulation of RCS from radar scenes: One of the most predictable technique for estimation of specific RCS of clutters is use of empirical models on the base of experimental results. As a main idea of empirical model is development of analytical solution (formula) for specific RCS in dependence from angle of the ray's incidence. Such kind of formulae is based on the regression analysis of big data of experimental results. As an example of modern empirical model is formula obtained by Wang et al. (2012) for specific RCS in centimeter wave range:

$$
\sigma^{0}=\gamma \cdot \mathrm{af}^{\mathrm{b}} \cdot \sin (\theta+\mathrm{c})=\mathrm{D} \cdot \sin (\theta+\mathrm{c})
$$

Where:

$\begin{array}{lll}\sigma^{0} & \text { Specific RCS of soil surface } \\ \mathrm{f} & : \text { Wave frequency } \\ \mathrm{a}, \mathrm{b}, \mathrm{c}, & : \text { Empirical coefficients for different } \\ \gamma \text { и } \mathrm{D} & \text { Kinds of soil surfaces } \\ \theta & : \text { Incident angle }\end{array}$

As a technique of description of sea clutters could be possible to consider specific RCS on the base of $\alpha$ distribution (Fiche et al., 2012). By Hui et al. (2013) the analytical model of sea surface's specific RCS is represented as a formula:

$$
\sigma_{6}^{0}=10 \lg \left(3,9.10^{-6} \lambda \psi^{0,4} \mathrm{G}_{\mathrm{a}} \mathrm{G}_{\mathrm{w}} \mathrm{G}_{\mathrm{u}}\right)
$$




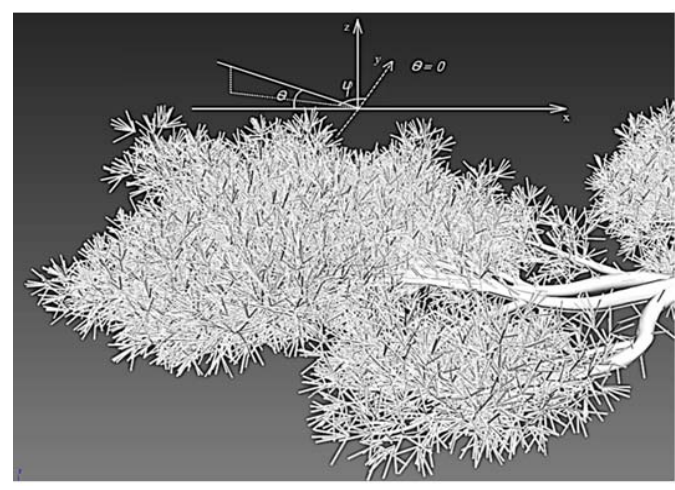

Fig. 1: Geometrical shape of pine on the base of cylinders

$\begin{array}{ll}\text { Where: } & \\ \sigma^{0} & : \text { Specific RCS of sea surface } \\ \lambda & \vdots \text { Wave length } \\ \psi & : \text { Angle of incidence } \\ \mathrm{G}_{\mathrm{a}}, \mathrm{G}_{\mathrm{w}}, \mathrm{G}_{\mathrm{u}}: & \text { Empirical coefficients in }\end{array}$

dependence of wind velocity. Each empirical model of specific RCS of clutter could be used only for limited kind of problems and with taking into account of experiment's conditions. So, no one empirical model couldn't be considered as universal for required clutter and frequency range.

One of the most difficult problem is simulation of scattering fields from plants because its geometric shape wouldn't be described analytically or with use of statistical techniques. Modern techniques are based on the description of trunks, branches, stalks and leaves as geometrical simple objects like cylinders or disks with simple RCS models. So, by Huang et al. (2015) characteristics of scattering of soy plants are investigated where the trunks and stalks were replaced with cylinders but leaves were replaced by flat disks. Reflection coefficients of trunk could be calculated on the infinite cylinder approximation. The applicability of a method of infinite cylinder approximation was checked by Dufva et al. (2009) and Thirion et al. (2004) as comparison with known Multi Level Fast Multi-pole Method (MLFMM). As an example the model of a branch of the pine simulated from cylindrical surfaces for evaluating her specific RCS is presented (Fig. 1).

Thickness of branches varied in a random way from 3-4 cm, thickness of needles-from 0,9-1 mm, length of needles from $4.1-5.3 \mathrm{~cm}$. Calculation was carried out (Dufva et al. 2009; Thirion et al., 2004) in the range of frequencies from 1,5-6 GHz. Also in case of calculation of plant's RCS such methods as $\mathrm{PO}, \mathrm{MoM}$ and an approximation method with discrete dipoles (Discrete Dipole Approximation) could be used (Liu et al., 2016; Le Toan et al., 1997; Koay et al., 2007). So, for example, by Koay et al. (2007) researches on reflection of electromagnetic waves from leaves of the parabolic form on the example of rice leaves were carried out. For the

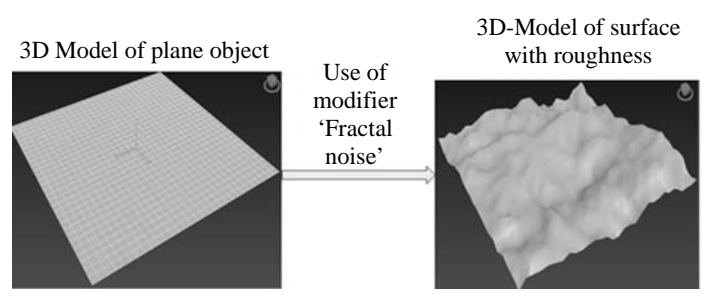

Fig. 2: Creation of surface with roughness in 3D Studio Max

problem of calculation of leaf's RCS with the parabolic form the method of approximation of Rayleigh-Hans is not acceptable (Rodriguez et al., 2016).

Described analytical methods of calculation of plant's RCS is suitable in rather narrow field of using. So, the approximation method in the form of the infinite cylinders considers only reflections from each separate cylinder without rereflections from other cylinders and surfaces (Thirion et al., 2004). The method of approximation of Rayleigh-Hans is suitable only for calculation of the fields reflected from narrow disks and also some other types of surfaces (cylinders, cones with the big relation of height to base radius) (Koay et al., 2007). PO is restricted to field of task problems in which the surface is provided by rather smooth geometrical primitives without sharp structures and also having the big electrical sizes, i.e., many big wavelengths.

To eliminate of short comings of empirical and analytical methods we could suggest an alternative method of calculation of the signal reflected from clutters on the basis of polygonal model of a radar scene and its multipoint model.

Technique of mathematical simulation of input signals of onboard radars: For digital simulation of radar scene's RCS the polygonal model of a scene as an array of plane triangles and edges were developed (Borzov et al., 2014; Suchkov et al., 2015). 3D Models of clutters as a base for its polygonal model could be created with use of 3D Studio Max. Process of creation of polygonal model with chaotic roughness in 3D Studio Max is based on imposing of the "fractal noise" modifier to an object. In Fig. 2, the process of creation of fractal surface is shown.

The 3D Models of radar targets could be developed in PTC Creo 4.0 (Borzov et al., 2014) as one of the most advanced software for simulation of complex shape surfaces. As a technique of creation of polygonal model of target in PTC Creo, we could suggest the using of Finite Elements Mesh (FEM) on the base of boundary Mesh application. The following step is exporting a mesh to PTCFEMNeutral Format (FNF File). With our special software, we could import mesh files from 3D Studio Max or PTC Creo to create digital polygonal model. As an example the polygonal model of Audi car is shown in Fig. 3. 


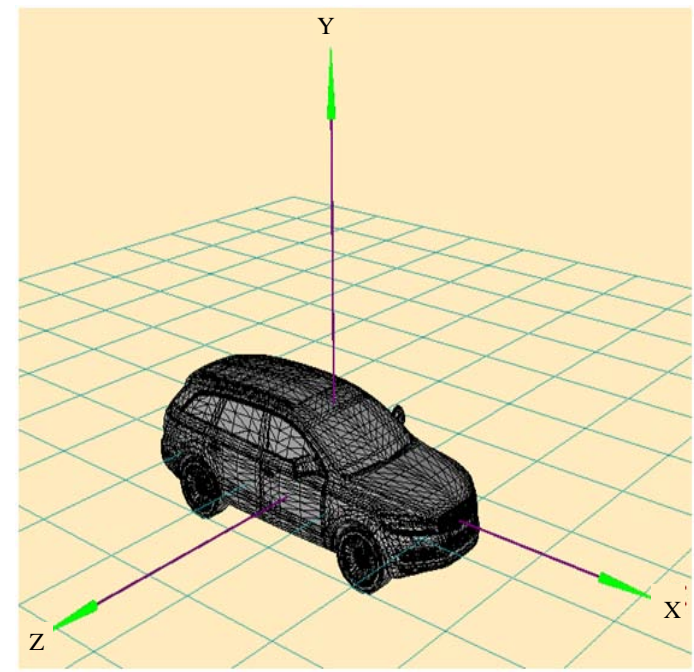

Fig. 3: Polygonal model of Audi car

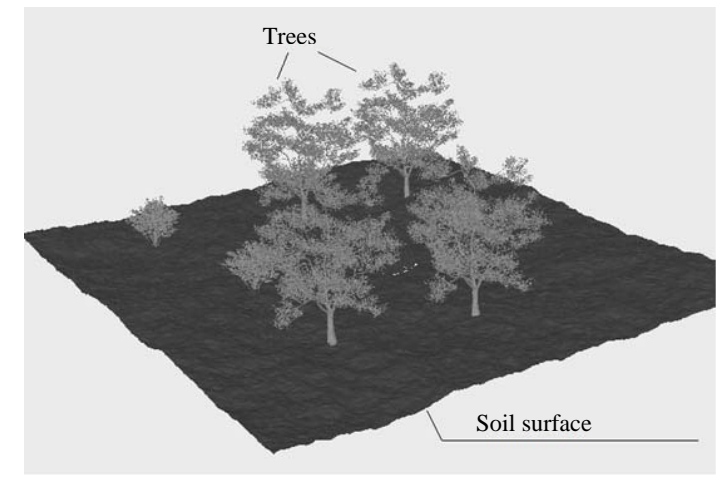

Fig. 4: Polygonal model of radar scene with trees and soil surface

Polygonal model of radar scene on the base of combination group of trees and soils roughness surface is shown in Fig. 4.

For creation of fast scattering model it would be possible to use arrays of RCS of different parallelepipeds, limited elements of radar scene (Likhoedenko and Suchkov, 2016). Multipoint model of a target consists of array of points of coordinates, array of RCS for each point in all directions (RCS diagram of local reflector) while each reflector (local point) is a center of parallelepiped limiting surface of a target. Process of transition to multipoint model from polygonal model demands considerable computing resources and can take some hours or days depending on complexity of geometry and quantity of primitives in polygonal model (Likhoedenko and Suchkov,2016). However, the ready multipoint model with the created RCS arrays allows with high speed to calculate the power of the signal reflected from a scene. As an example, the multipoint model of Audi car in Fig. 5 is shown.

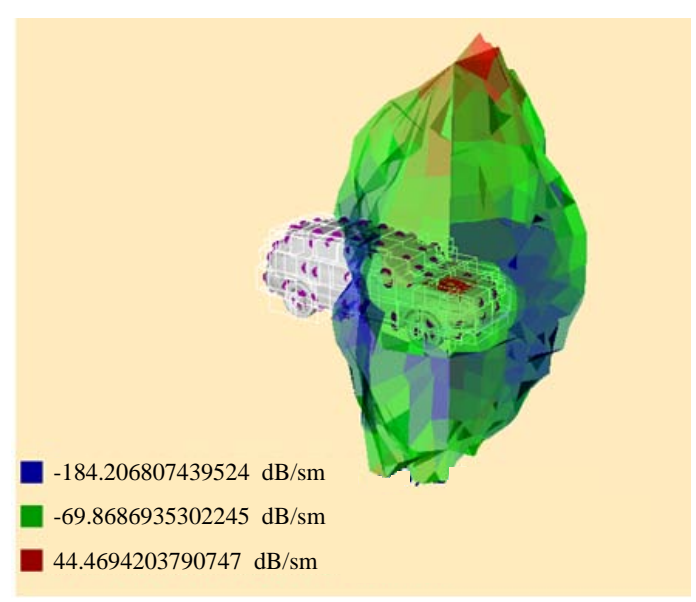

Fig. 5: Multipoint model of car Audi with RCS diagram of local reflector

The power of an input signal of onboard radar could be calculated on the base of multipoint model by a formula (Likhoedenko and Suchkov, 2016):

$$
\mathrm{P}=\frac{\mathrm{P}_{\mathrm{i}} \mathrm{P}_{\mathrm{i}} \mathrm{D}_{\mathrm{s}} \lambda^{2}}{(4 \pi)^{2}}\left|\sum_{\mathrm{k}=1}^{\mathrm{N}_{\mathrm{m}}} \frac{\mathrm{F}_{\mathrm{ik}} \mathrm{F}_{\mathrm{se}} \mathrm{P}_{\mathrm{k}}}{\mathrm{R}_{\mathrm{k}}^{2}}\right|^{2}
$$

Where:

$\mathrm{Nm}$ : Number of local reflectors of multipoint model

$\rho_{\mathrm{k}} \quad$ : Complex coefficient of reflection from local point

$P_{i} \quad$ : Power of transmitter of radar

$\mathrm{D}_{\mathrm{i}(\mathrm{s})}$ : Gain of transmitting (receiving) antenna of radar

$\lambda^{1(s)}$ : Wave length

$\mathrm{R}_{\mathrm{k}}$ : Distance from $\mathrm{k}$-th point of model to radar

$\mathrm{F}_{\mathrm{i}(\mathrm{s}) \mathrm{k}}$ : Pattern of transmitting (receiving) antenna in direction of $\mathrm{k}$-th point of model

The complex coefficient of reflection from local point of multipoint model is defined from formula:

$$
\rho_{k}=\sqrt{\frac{\sigma_{k}}{4 \pi} \exp \left(-\mathrm{i} 2 \frac{2 \pi}{\lambda} \mathrm{R}_{\mathrm{k}}\right)}
$$

Where, $\sigma$ RCS of k-th point from calculated diagram.

\section{RESULTS AND DISCUSSION}

Technique of mathematical simulation of input signals of onboard radars: Also, as an example, the calculation of the signal reflected from the radar scene of the forest area is shown. The model of the scene of the forest area represents combination of polygonal model of a soil surface and polygonal model of five trees (Fig. 4). The situation of flight over the radar scene of the Unmanned Aerial Vehicle (UAV) with onboard radar was simulated (Fig. 6-9). 

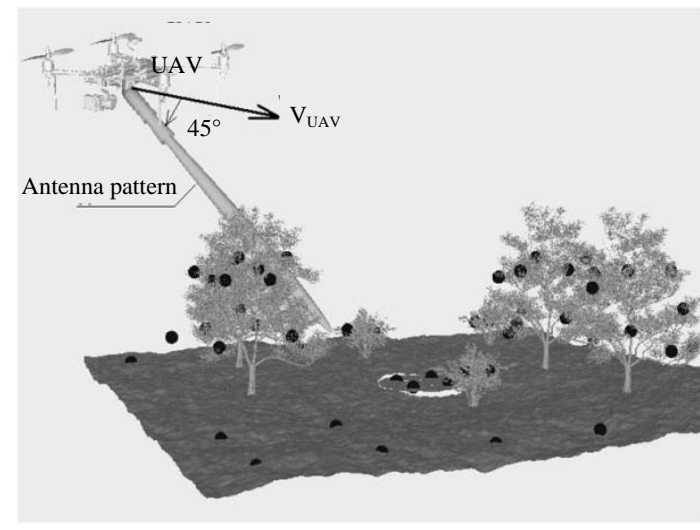

Fig. 6: The scheme of a radar-location of the radar scene with trees presented by multipoint model by UAV's radar
Height of flight over the earth was $10 \mathrm{~m}$, a flight distance was $25 \mathrm{~m}$. In Fig. 10, results of calculation of power of an input signal of the onboard radar (3) reflected from this scene are shown. Soil parameters: the relative dielectric permittivity $\varepsilon=5$, conductivity $\sigma=0,2 \mathrm{Cmm}^{-1}$, trunks: $\varepsilon=2.5, \sigma=0.5 \mathrm{Cmm}^{-1}$; leaves: $\varepsilon=5.5, \sigma=2$ $\mathrm{Cmm}^{-1}$. In Fig. 6, the dependence of signal power on horizontal range is shown. Parameters of onboard radar was established the following: wavelength $\lambda \sim 3 \mathrm{~cm}$, transmitter power $\sim 10 \mathrm{wt}$., antenna gain was 10 , antenna pattern width $\Delta \theta \sim 5^{\circ}$.

So, the developed model allows to define the signal reflected from trees that can be used for detection of the objects located on the scene with the forest area.

As an example of use of multipoint model the simulation of input signals of automotive radar with the following parameters was carried out: $\mathrm{P}_{\mathrm{i}}=10 \mathrm{mWt}$, $\operatorname{Di}(\mathrm{s})=400, \mathrm{f}_{0}=77 \mathrm{GHz}$, width of the main lobe of antenna pattern is $25^{\circ}$. The simulation of an input signal

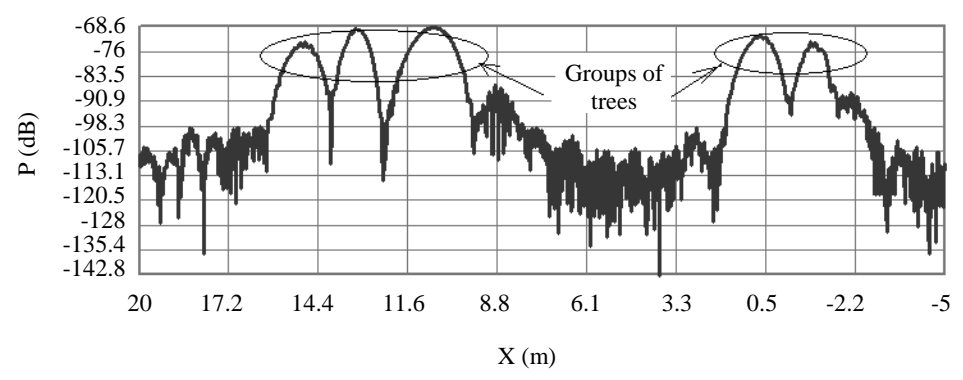

Fig. 7: Power of an input signal of the onboard radar reflected from the scene with trees
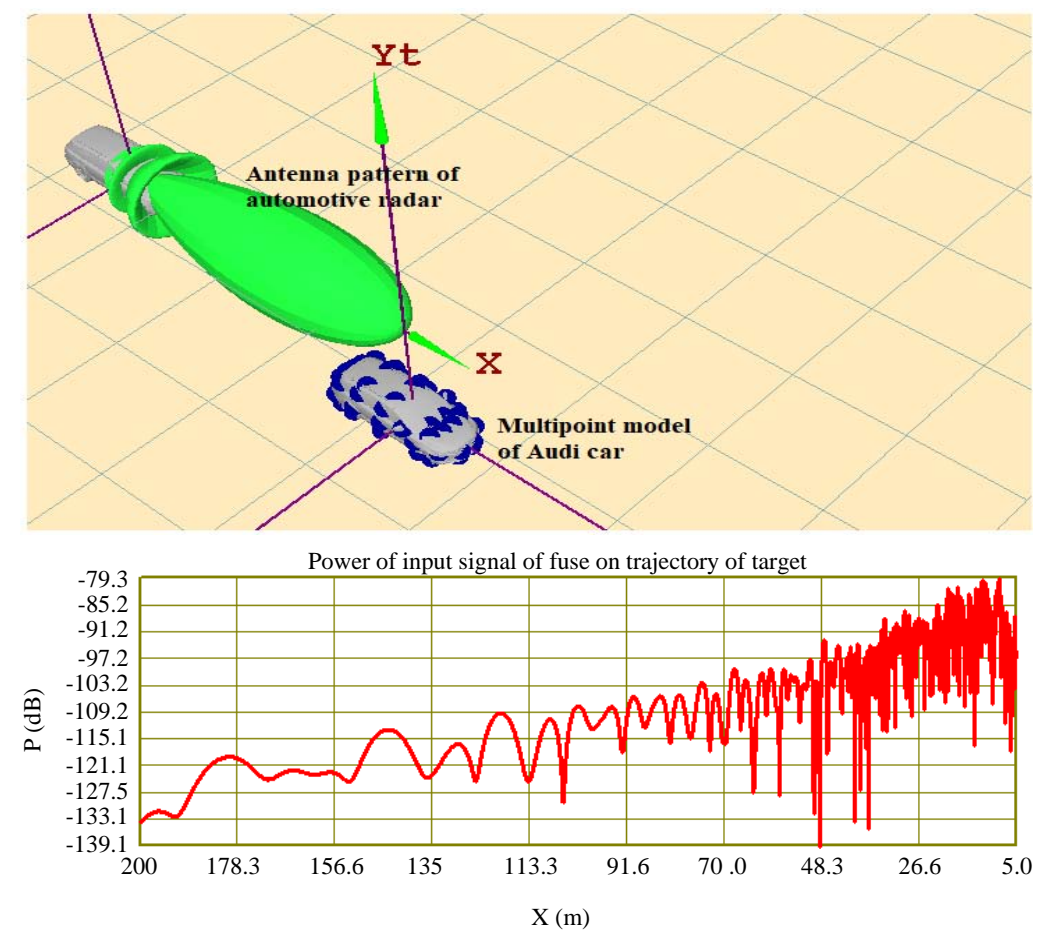

Fig. 8: Results of simulation of input signal of automotive radar from car on the trajectory of movement 


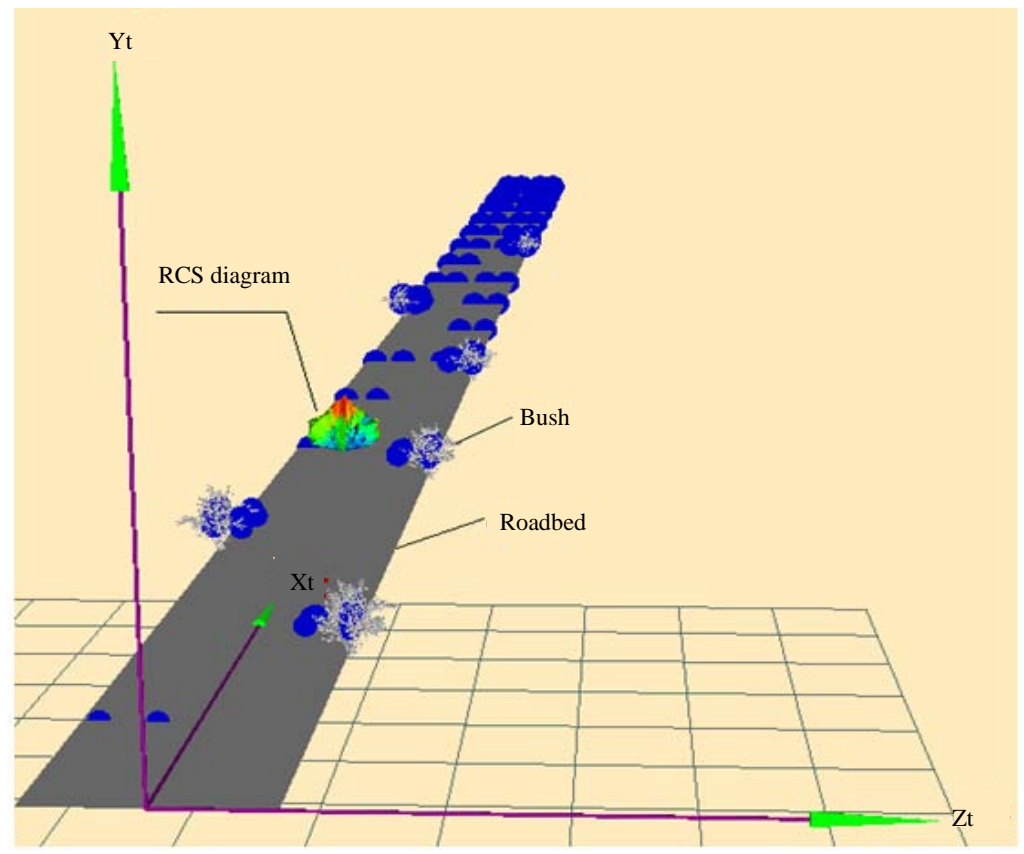

Fig. 9: Multipoint model of roadbed in frequency $77 \mathrm{GHz}$

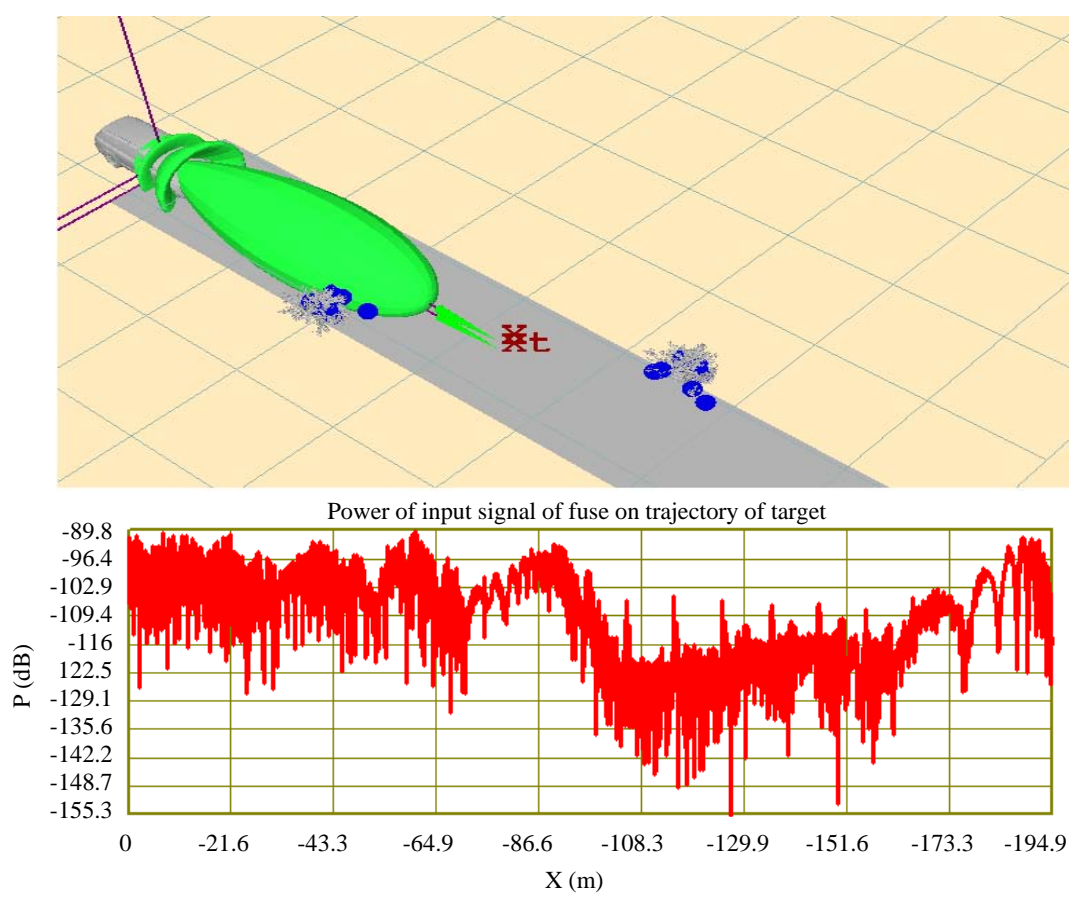

Fig. 10: Results of simulation of input signal of automotive radar from roadbed on the trajectory of movement

of automotive radar was carried out on a direct trajectory in the range of horizontal distances $\mathrm{X}=200.5$ m Fig. 7.

The multipoint models of the roadbed with RCS diagram of one of points calculated on frequency of the radar $\mathrm{f}=77 \mathrm{GHz}$ is shown in Fig. 8 .
For estimation of influence of reflections from a roadbed and local objects the simulation of an input signal of the radar from the roadbed in the range of horizontal ranges $X=200.5 \mathrm{~m}$ (Fig. 9) has been carried out. Also, the dependence of power of an input signal of automotive radar on a roadbed and bushes is shown in Fig. 9. 


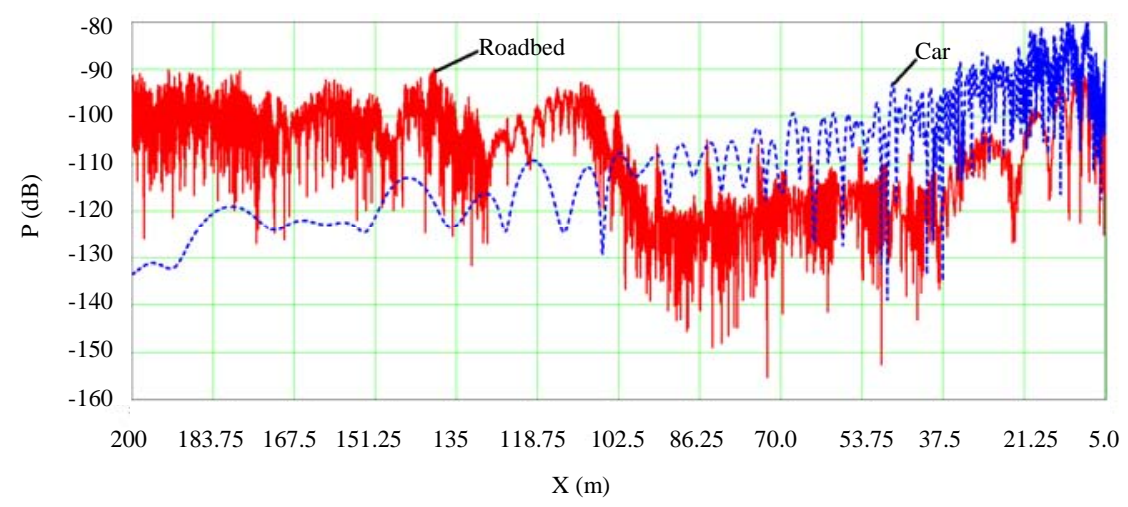

Fig. 11: Dependence of power of an input signal of automotive radar on the moving car (shaped curve) and on a roadbed (continuous curve) at frequency of $77 \mathrm{GHz}$

Comparison of diagrams of power of an input signals of automotive radar from the car and from the roadbed is shown in Fig. 10.

So, in the frequency range of $77 \mathrm{GHz}$ the power of the reflected signal from local objects and roadbed much more higher than the level of a signal from the car at distances of $100.200 \mathrm{~m}$ from the automotive radar (Fig. 11).

\section{CONCLUSION}

The developed method of simulation of input signals of o nboard radars from the radar scene with clutters is the effective technology of improving of algorithms of detection and selection of the target in trajectory signal. Application of the software with multipoint model constructed on the basis of polygonal model of targets and clutters allows to avoid the short comings inherent in the considered analytical and empirical methods of calculation of the signal reflected by clutter of a nonuniform geometrical configuration. The multipoint model allows to calculate of input signals of the UAV radio altimeter or automotive onboard radar reflected from clutters practically of any complexity regardless of the nature of roughnesses, statistical and physical characteristics of clutter. The main complexity of practical use of multipoint model consists in need of existence of full information on electrophysical structure of radar scene and clutters.

\section{REFERENCES}

Borzov, A.B., V.B. Suchkov, B.I. Shakhtarin and Y.A. Sidorkina, 2014. Mathematical modeling and simulation of the input signals of short-range radar systems. J. Commun. Technol. Electron., 59: $1356-1368$.

Dufva, T., J. Praks, S. Jarvenpaa and J. Sarvas, 2009. Scattering model for a pine tree employing VIE with a broadband MLFMA and comparison to ICA. IEEE. Trans. Geosci. Remote Sens., 48: 1119-1127.
Fiche, A., A. Khenchaf, J.C. Cexus, M. Rochdi and A. Martin, 2012. RCS characterization of sea clutter by using the $\alpha$-stable distributions. IEEE. Trans. Geosci. Remote Sens., 41: 55-61.

Huang, H., S.B. Kim, L. Tsang, X. Xu and T.H. Liao et al., 2015. Coherent model of L-band radar scattering by soybean plants: Model development, evaluation and retrieval. IEEE. J. Sel. Top. Appl. Earth Obs. Remote Sens., 9: 272-284.

Hui, T., W. Ding'e and Y. Xuanyuan, 2013. Simulation of impact of sea clutter to radar detecting performance. Proceedings of the 2013 IEEE 5th International Symposium on Microwave, Antenna, Propagation and EMC Technologies for Wireless Communications, October 29-31, 2013, Chengdu, China, ISBN:978-1-4673-6077-7, pp: 505-510.

Koay, J.Y., C.P. Tan, K.S. Lim, S.B. bin Abu Bakar and H.T. Ewe et al., 2007. Paddy fields as electrically dense media: Theoretical modeling and measurement comparisons. IEEE. Trans. Geosci. Remote Sens., 45: 2837-2849.

Le Toan, T., F. Ribbes, L.F. Wang, N. Floury and K.H. Ding et al., 1997. Rice crop mapping and monitoring using ERS-1 data based on experiment and modeling results. IEEE. Trans. Geosci. Remote Sens., 35: 41-56.

Likhoedenko, A.K. and V.B. Suchkov, 2016. Development of fast scattering model of complex shape target for seminatural tests of onboard proximity radars in real time mode. Proceedings of the International Conference on Frontiers of Sensors Technologies (ICFST 2016) Vol. 59, March 12-14, 2016, Hong Kong, China, ISBN:9781510824652, pp: $1-4$.

Liu, L., Y. Shao, K. Li and Z. Yang, 2016. Modelling microwave backscattering from parabolic rice leaf. IEEE. Trans. Geosci. Remote Sens., 35: 3676-3679. 
Rodriguez, I., R. Abreu and P.L. Erika, 2016. The 24 $\mathrm{GHz}$ CMWAVE radio propagation through vegetation: Suburban tree clutter attenuation. Proceedings of the 2016 10th European Conference on Antennas and Propagation (EuCAP), April 10-15, 2016, IEEE, Davos, Switzerland, pp: 1-5.

Suchkov, V.B., M.I. Baev and A.K. Likhoedenko, 2015. Development of parallel algorithms for calculation of backscattering characteristics of complex shape objects. Proceedings of the 5th International Workshop on Computer Science and Engineering (WCSE 2015), April 17-19, 2015, Bauman, Moscow, pp: 103-108.
Thirion, L., I. Chenerie and C. Galy, 2004. Application of a coherent model in simulating the back scattering coefficient of a mangrove forest. Waves Random Media, 14: S299-S316.

Wang, X., H. Wang, S. Yan, L. Li and C. Meng, 2012. Simulation for surveillance radar ground clutter at low grazing angle. Proceedings of the 2012 International Conference on Image Analysis and Signal Processing, November 9-11, 2012, IEEE, Hangzhou, China, ISBN: 978-1-4673-2547-9, pp: 1-4. 